EPJ Web of Conferences 113,01001 (2016)

DOI: $10.1051 /$ epjconf/20161130100 1

(C) Owned by the authors, published by EDP Sciences, 2016

\title{
Hadron Resonances from QCD
}

\author{
Jozef J. Dudek ${ }^{1,2, a}$ for the Hadron Spectrum Collaboration \\ ${ }^{1}$ Department of Physics, Old Dominion University, Norfolk, VA 23529, USA \\ ${ }^{2}$ Theory Center, Jefferson Lab, 12000 Jefferson Avenue, Newport News, VA 23606, USA.
}

\begin{abstract}
I describe how hadron-hadron scattering amplitudes are related to the eigenstates of QCD in a finite cubic volume. The discrete spectrum of such eigenstates can be determined from correlation functions computed using lattice QCD, and the corresponding scattering amplitudes extracted. I review results from the Hadron Spectrum Collaboration who have used these finite volume methods to study $\pi \pi$ elastic scattering, including the $\rho$ resonance, as well as coupled-channel $\pi K, \eta K$ scattering. The very recent extension to the case where an external current acts is also presented, considering the reaction $\pi \gamma^{\star} \rightarrow \pi \pi$, from which the unstable $\rho \rightarrow \pi \gamma$ transition form factor is extracted. Ongoing calculations are advertised and the outlook for finite volume approaches is presented.
\end{abstract}

\section{Introduction}

Quantum Chromodynamics presents us with a unique challenge within the Standard Model - the observed spectrum of strongly interacting particles, hadrons, does not appear to trivially follow from the basic quark and gluon fields whose interactions are described by the QCD lagrangian. Indeed the observed experimental spectrum shows a degree of regularity yet to be explained from first principles, and, to date, seems to lack states one might expect within QCD where the gluonic field plays an active role: glueballs and hybrid mesons.

The excited spectroscopy of QCD is the study of resonances - excited hadrons are not asymptotic states, they decay rapidly to lighter stable hadrons, and appear in experiments as enhancements in the scattering of these stable hadrons. It follows that to rigorously study the excited spectrum of QCD we should calculate scattering amplitudes and examine their resonant content.

Lattice QCD is a first-principles approach to the theory which considers the quark and gluon fields on a finite discretized grid of points, computing correlation functions as the average over a set of Monte-Carlo generated field configurations. The spectrum of eigenstates for example can be determined by considering the Euclidean time dependence of two-point correlation functions using operators having the quantum numbers of hadrons, constructed from quark and gluon fields. Lattice QCD is thus a controlled approximation in which the artificially introduced scales, the lattice spacing and the lattice volume, can be varied, and ultimately the true theory reached in the appropriate limits. In practice many calculations also opt to work with values of the light-quark mass parameter larger than that found experimentally, thus reducing the computational cost.

\footnotetext{
ae-mail: dudek@jlab.org
} 
By necessity Lattice QCD calculations are done in a finite Euclidean volume, and in such a box, where asymptotic states cannot be prepared, there is no scattering in the usual sense, so it is not immediately obvious how one is to go about computing scattering amplitudes. Fortunately, it is the case that the discrete spectrum of states in a finite volume is determined by the scattering amplitudes, and by computing the spectrum in one or more volumes, we may hope to determine these scattering amplitudes. Then by examining the energy dependence of these amplitudes, we may find their resonant content and make statements about the excited spectrum of QCD.

\section{Scattering amplitudes \& the spectrum in a finite volume}

The effect of a finite periodic boundary on the spectrum of eigenstates of a quantum system can be illustrated by considering non-relativistic quantum mechanics in one space dimension. Free particles of definite momentum are described by plane-wave wavefunctions, $e^{i p x}$, and application of a periodic boundary condition at $x=L$ indicates that free-particle momentum must be quantized in units of $\frac{2 \pi}{L}$. For two identical particles interacting via an arbitrary finite-range potential, applying the boundary condition to a scattering solution outside the range of the potential, $\cos (p|x|+\delta(p))$, leads to a condition $p=\frac{2 \pi}{L}-\frac{2}{L} \delta(p)$, which determines the allowed discrete values of momentum (and hence energy) in terms of the elastic scattering phase shift, $\delta$. It follows that the discrete spectrum at any given volume, $L$, contains information about the scattering amplitude in infinite volume.

The more complicated case of elastic scattering in a quantum field theory in $3+1$ dimensions, having a cubic periodic boundary, was worked out by Lüscher for the rest frame, and extensions to moving frames appeared later [1-5]. Conceptually the result is similar to that above, with some slight complications arising because the cubic symmetry of the boundary is not commensurate with the angular-momentum basis we use to characterize partial waves. These complications can be handled by considering the reduced symmetry group of the boundary, at rest or boosted, which has a finite number of irreducible representations.

Going beyond elastic scattering, the formalism for coupled-channel scattering has been derived independently in a number of differing approaches to field theory [6-9], all leading to the same condition relating the scattering $t$-matrix to the discrete spectrum of states in a finite volume:

$$
\operatorname{det}\left[\mathbf{t}^{-1}(E)+i \rho(E)-\mathbf{M}(E, L)\right]=0
$$

The determinant is over the space of open scattering channels ${ }^{1}$, and $\rho(E)$ is the phase space matrix for these channels, with $\mathbf{M}(E, L)$ being a matrix of known, essentially kinematic, functions. The discrete values of $E(L)$ which solve this equation provide the spectrum of QCD in this finite $L \times L \times L$ volume. It follows that a first-principles calculation of this spectrum in one or more volumes could yield information about $\mathbf{t}(E)$, and we turn our attention to lattice QCD as the tool to determine the spectrum.

\section{Determining the spectrum using lattice QCD}

Two-point Euclidean correlation functions have a spectral representation,

$$
\left\langle 0\left|O_{i}(t) O_{j}^{\dagger}(0)\right| 0\right\rangle=\sum_{\mathfrak{n}} e^{-E_{n} t}\left\langle 0\left|O_{i}\right| \Uparrow\right\rangle\left\langle 0\left|O_{j}\right| \Uparrow\right\rangle^{*}
$$

\footnotetext{
${ }^{1}$ and over the partial waves subduced into the particular irreducible representation under consideration
} 
where the discrete spectrum of eigenstates of QCD having the quantum numbers of the operators $O_{i, j}$ appears. These operators should be constructed from the basic discretized quark and gluon fields of lattice QCD, and ideally to determine the spectrum efficiently we would like to make the values of $\left\langle 0\left|O_{i, j}\right| \mathfrak{n}\right\rangle$ as large as possible for the low-lying states we are interested in. This can be achieved by developing operators which 'closely resemble' the eigenstates.

A set of operators which have been shown to efficiently interpolate 'single-meson' states [1013] take the form $\bar{\psi} \Gamma \overleftrightarrow{D} \ldots \overleftrightarrow{D} \psi$, i.e. a (smeared) quark bilinear featuring Dirac gamma structure and some number of gauge-covariant derivatives. Operators resembling a pair of mesons can be constructed as products of the bilinears presented above, e.g. $\sum_{\vec{p}_{1} \vec{p}_{2}} C\left(\vec{p}_{1}, \vec{p}_{2}\right) \pi\left(\vec{p}_{1}\right) \pi\left(\vec{p}_{2}\right)$ where $\pi(\vec{p}) \sim \sum_{\vec{x}} e^{i \vec{p} \cdot \vec{x}} \vec{\psi} \Gamma \psi(\vec{x})$ where $\boldsymbol{\Gamma}$ is such that the bilinear has pseudoscalar quantum numbers and overlaps strongly with the ground state pion. The generalized Clebsch-Gordan coefficients, $C\left(\vec{p}_{1}, \vec{p}_{2}\right)$, are chosen to ensure that the operator transforms in the desired irreducible representation of the reduced rotational group.

Calculation of correlation functions using these operators can be done in an efficient manner using the distillation technology first presented in Ref. [14]. A spectrum of excited levels can be extracted from a matrix of correlation functions, computed using a basis of operators of the type described above. The variational best estimate for the spectrum is obtained by solving the generalized eigenproblem [15-17],

$$
\mathbf{C}(t) \boldsymbol{v}^{\mathfrak{n}}=\lambda_{\mathfrak{n}}(t) \mathbf{C}\left(t_{0}\right) \boldsymbol{v}^{\mathfrak{n}},
$$

where the eigenvalue $\lambda_{\mathfrak{n}}(t) \rightarrow e^{-E_{\mathfrak{n}}\left(t-t_{0}\right)}$ supplies us with the energy of the $\mathfrak{n}^{\text {th }}$ state.

\section{Elastic $\pi \pi$ scattering and the $\rho$ resonance}

One of the simplest scattering problems in hadron physics is $\pi \pi$ in isospin-2, where experimentally there are found to be no low-lying resonances. The minimal quark content of this channel is $u u \bar{d} \bar{d}$ and a suitable basis of operators resembles pairs of operators capable of interpolating pions: $\sum_{\vec{p}_{1} \vec{p}_{2}} C\left(\vec{p}_{1}, \vec{p}_{2}\right) \pi\left(\vec{p}_{1}\right) \pi\left(\vec{p}_{2}\right)$. The Hadron Spectrum Collaboration computed [13] finite volume energy spectra in a calculation where the light-quark masses took a value such that $m_{\pi}=391 \mathrm{MeV}$. The spectra were obtained on three different volumes and in several different moving frames. Using the elastic Lüscher formalism and its moving frame extensions, phase shift values spanning the entire elastic region (below $4 \pi$ threshold) for $S$ and $D$ waves were obtained as presented in Figure 1 . The results show the same qualitative features as experiment, that of a weakly repulsive interaction which is strongest in $S$-wave.

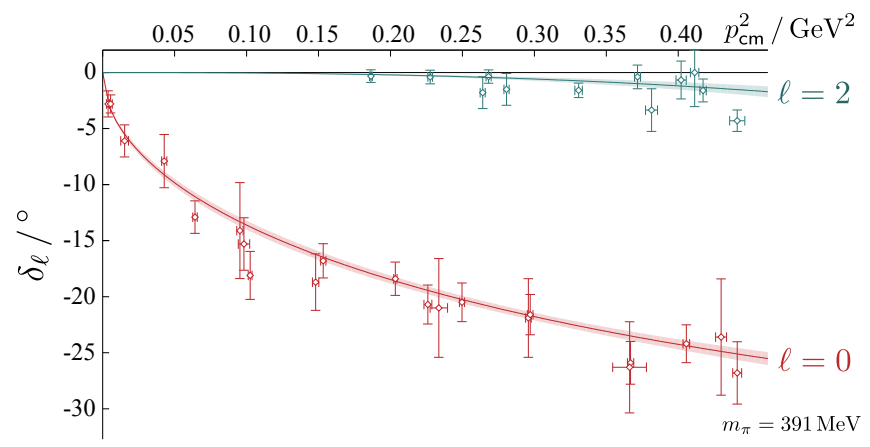

Figure 1. Elastic scattering phase shift in degrees for isospin-2 $\pi \pi$ in $S$-wave and $D$-wave plotted as a function of cm-frame momentum. Scattering length parameterizations describe the energy dependence. Computed with quark masses corresponding to a pion mass of 391 $\mathrm{MeV}$. Figure taken from [13]. 
A slightly less simple scattering problem is $\pi \pi$ in isospin-1, where on the basis of experiment, we expect a vector resonance, the $\rho$. A suitable operator basis for the computation of correlation functions supplements the " $\pi \pi$-like" operators presented previously with $\bar{\psi} \mathbf{\Gamma} \psi$ operators likely to overlap well with a tightly-bound $q \bar{q}$ state (as we expect the $\rho$ to dominantly be). Computation [18] on the same set of lattices as described above leads to the elastic scattering phase shift presented in Figure 2 . The energy dependence of the scattering amplitude can be described well by a relativistic Breit-Wigner form corresponding to a single isolated resonant state. The small width $\Gamma \sim 12 \mathrm{MeV}$ of the extracted resonance is a result of the small phase space for decay of an $855 \mathrm{MeV}$ resonance into two $391 \mathrm{MeV}$ pions. If we define a $\rho \rightarrow \pi \pi$ coupling via $\Gamma=\frac{g_{R}^{2}}{6 \pi} \frac{p^{3}}{m_{R}^{2}}$, the extracted coupling has value $g_{R}=5.70(11)$, which is compatible with the experimental value [19] of $\sim 6.0(1)$, in line with the expectation that it is almost quark mass independent [20], and only the phase space is changing as the pion mass decreases to its physical value.

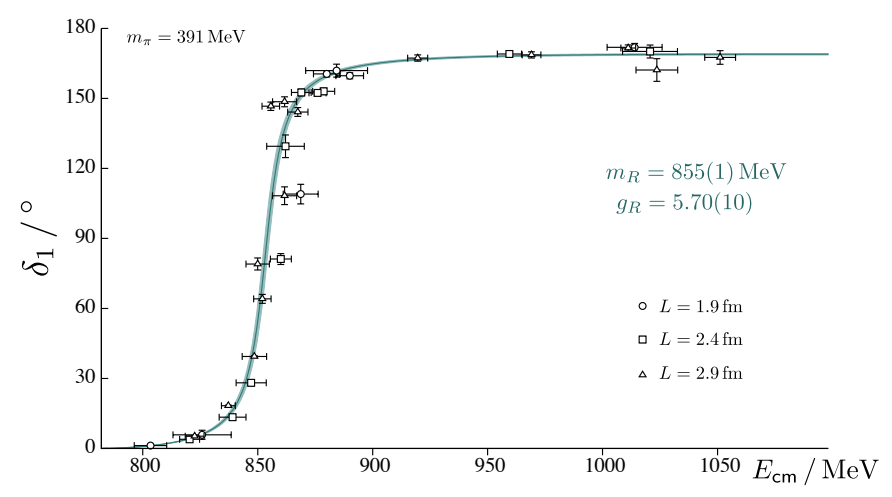

Figure 2. Elastic scattering phase shift in degrees for isospin- $1 \pi \pi$ in $P$-wave plotted as a function of $\mathrm{cm}$-frame energy. A relativistic Breit-Wigner parameterization describes the energy dependence. Computed with quark masses corresponding to a pion mass of 391 $\mathrm{MeV}$. Figure taken from [18].

A recent calculation $[21]^{2}$ performed with lighter quark masses such that $m_{\pi}=236 \mathrm{MeV}$ is presented in Figure 3 where we observe the expected increase in width, decrease in $\rho$ mass, and negligible change in $g_{R}$.

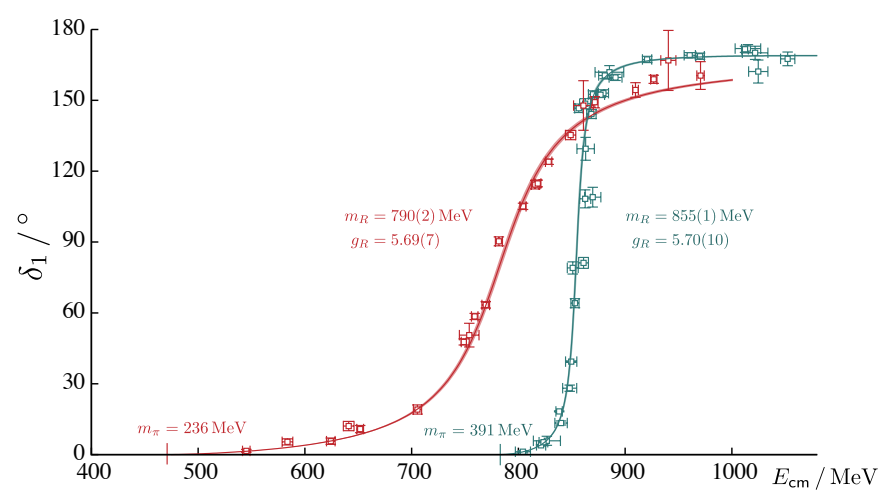

Figure 3. Elastic scattering phase shifts in degrees for isospin-1 $\pi \pi$ in $P$-wave plotted as a function of $\mathrm{cm}$-frame energy. Comparison of calculations performed with quark masses corresponding to pion masses of $391 \mathrm{MeV}$ and $236 \mathrm{MeV}$. Figure taken from [21].

\footnotetext{
${ }^{2}$ this calculation also considered coupled channel amplitudes into $K \bar{K}$, finding essentially no coupling at low energies.
} 


\section{Coupled-channel $\pi K, \eta K$ scattering}

Refs [22, 23] from the Hadron Spectrum Collaboration present the first lattice QCD calculation to extract coupled-channel scattering amplitudes, considering the strangeness-1, isospin-1/2 channel, which at low energies features $\pi K$ and $\eta K$ scattering. Spectra were extracted from correlation functions computed with operators resembling $\pi K\left(\sum_{\vec{p}_{1} \vec{p}_{2}} C\left(\vec{p}_{1}, \vec{p}_{2}\right) \pi\left(\vec{p}_{1}\right) K\left(\vec{p}_{2}\right)\right), \eta K$ $\left(\sum_{\vec{p}_{1} \vec{p}_{2}} C\left(\vec{p}_{1}, \vec{p}_{2}\right) \eta\left(\vec{p}_{1}\right) K\left(\vec{p}_{2}\right)\right)$ and $q \bar{q}$-like constructions $(\bar{\psi} \mathbf{\Gamma} \psi)$. Some example spectra are shown in Figure 4 where we see that a large number of energy levels can extracted, even in cases of approximate degeneracy, with good statistical precision.
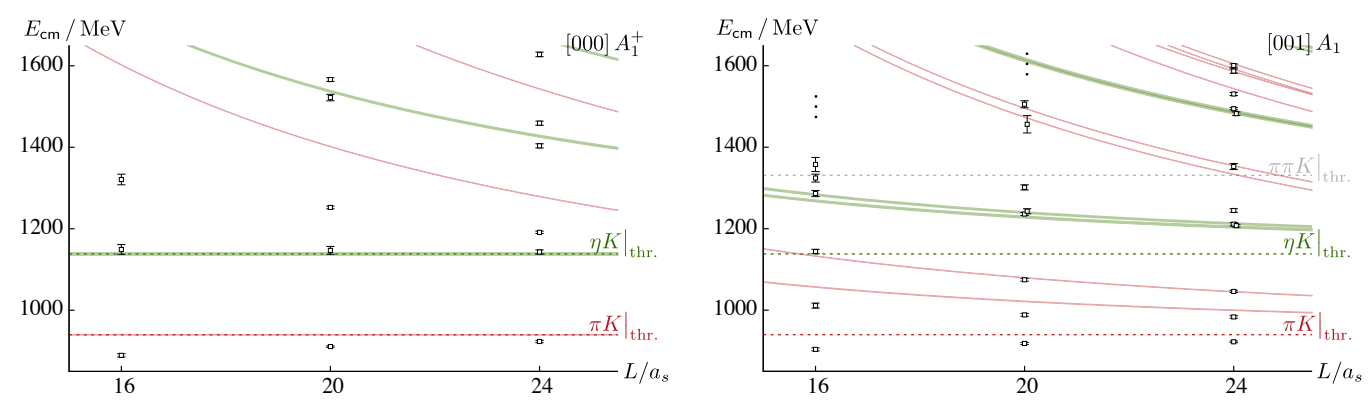

Figure 4. Spectrum of QCD eigenstates with strangness-1 and isospin-1/2 extracted from lattice QCD calculations on three volumes $\left(16^{3}, 20^{3}, 24^{3}\right)$ with quark masses such that $m_{\pi}=391 \mathrm{MeV}$. (a) Rest frame spectrum in $A_{1}^{+}$irrep (dominated by $J^{P}=0^{+}$). (b) Spectrum in a frame moving with one unit of lattice momentum in the irrep $A_{1}$. Red, green lines show the positions of non-interacting $\pi K, \eta K$ energy-levels respectively. Figure taken from $[22,23]$.

Extraction of the coupled-channel $t$-matrix from these levels is challenging. At any given energy the unitarity preserving, time-reversal invariant $t$-matrix is described by $\frac{1}{2} N(N+1)$ real numbers. This means that Equation 1, which relates the finite volume spectrum to the $t$-matrix features $\frac{1}{2} N(N+1)$ unknowns for each energy level and this clearly cannot be solved on a level-by-level basis.

An approach to get around this problem is to parameterize the energy dependence of the $t$-matrix and to attempt to describe the entire spectrum of states, even in multiple different volumes, by varying parameters until the best match to the lattice QCD spectrum is obtained. This was done in [22, 23], trying a varied set of parameterizations based upon the $K$-matrix, and consistent results for the energy dependence of the amplitudes were obtained. An example is presented in Figure 5 for $S$-wave, $P$-wave and $D$-wave scattering, where the amplitudes are expressed in terms of phase shifts and an inelasticity parameter defined below:

$$
t_{\pi K, \pi K}=\frac{1}{2 i \rho_{\pi K}}\left(\eta e^{2 i \delta_{\pi K}}-1\right), t_{\eta K, \eta K}=\frac{1}{2 i \rho_{\eta K}}\left(\eta e^{2 i \delta_{\eta K}}-1\right), t_{\pi K, \eta K}=\frac{1}{2 \sqrt{\rho_{\eta K} \rho_{\pi K}}}\left(\sqrt{1-\eta^{2}} e^{i\left(\delta_{\pi K}+\delta_{\eta K}\right)}\right)
$$

The $\pi K P$-wave amplitude is found to feature a bound state only slightly below threshold - that this $K^{\star}$ does not appear as a resonance as it does in experiment is a consequence of the quark mass used, for any smaller light quark mass, the state would appear above threshold with a decay width into $\pi K$. 

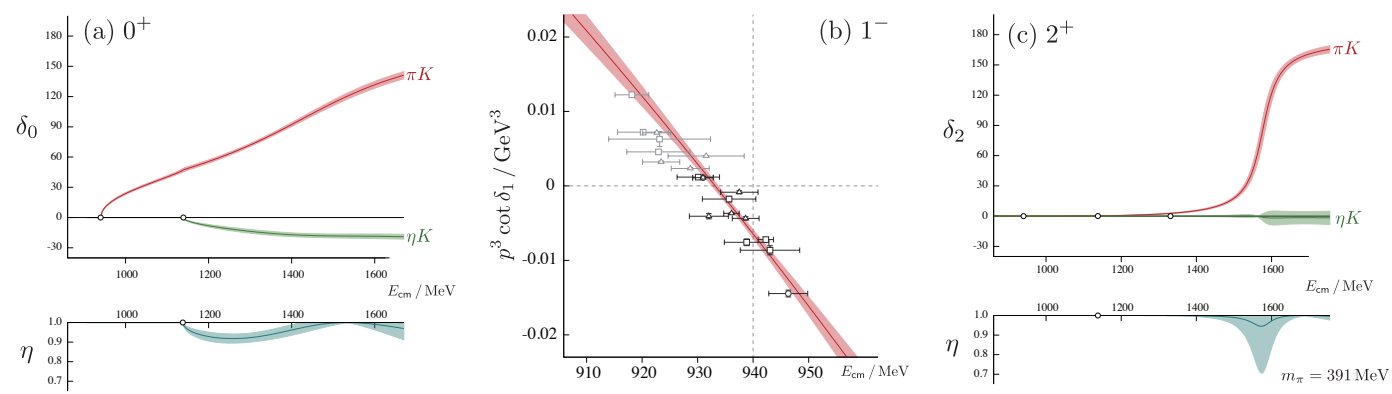

Figure 5. Coupled channel $\pi K, \eta K$ amplitudes determined at $m_{\pi}=391 \mathrm{MeV}$. (a) Phase shifts and inelasticity in $J^{P}=0^{+}$partial wave. (b) $\pi K$ phase shift for $J^{P}=1^{-}$in threshold region - zero crossing of $p^{3} \cot \delta_{1}$ corresponds to a vector bound state. (c) Phase shifts and inelasticity in $J^{P}=2^{+}$partial wave - rapid increase in $\pi K$ phase indicative of a narrow tensor resonance, essentially decoupled from the $\eta K$ channel. Figure taken from [22, 23].

The $S$-wave $t$-matrix shows only very weak coupling between the $\pi K$ and $\eta K$ channels. The gradual increase in the $\pi K$ phase shift around $1400 \mathrm{MeV}$ corresponds to a pole singularity far into the complex plane which may be identified with a broad scalar resonance. The experimental $K_{0}^{\star}(1430)$, which decays to $\pi K$ and not $\eta K$ may be related to this state. Another $S$-wave singularity is also present causing the relatively rapid rise in phase shift at $\pi K$ threshold. This pole lies on the real axis, below the threshold on the unphysical sheet and is thus considered a virtual bound state. Suggestions from unitarized chiral perturbation theory are that the experimental resonance singularity known as the $\kappa$ may become just such a virtual bound state as the mass of the pion is increased [20].

The determination of the $J^{P}=2^{+}$amplitude is somewhat less rigorous owing to the neglect of the $\pi \pi K$ channel in this first calculation. Nevertheless a consistent description of the finite volume spectrum can be obtained considering only $\pi K$ and $\eta K$ scattering, and the extracted amplitude shows decoupling of the two channels and a clear narrow tensor resonance decaying to $\pi K$. This state is likely related to the experimental $K_{2}^{\star}(1430)$.

\section{$6 \pi \gamma^{\star} \rightarrow \pi \pi$ and the unstable $\rho$ transition form factor}

An extension of the finite volume formalism to deal with the case where external currents are present (building upon the ideas outlined in [24]) has recently appeared [25, 26], suggesting that the finite volume matrix elements of the current which can be extracted from three-point correlation functions can be related to their infinite volume counterparts.

The development of the distillation technology needed to efficiently calculate the required threepoint correlation functions in the case of the vector current relevant to electromagnetic amplitudes was presented in [27], and the first calculation of an electromagnetic scattering amplitude, $\pi \gamma^{\star} \rightarrow \pi \pi$, has very recently appeared [28]. In this paper, the Hadron Spectrum Collaboration determined matrix elements of the type $\left\langle\pi\left|\bar{\psi} \gamma_{\mu} \psi\right| \pi \pi\right\rangle$ on a lattice of volume $\sim(2.4 \mathrm{fm})^{3}$ with $m_{\pi}=391 \mathrm{MeV}$ and from these extract the scattering amplitude illustrated in Figure 6. The $\rho$ resonance which couples to both $\gamma \pi$ and $\pi \pi$ clearly dominates the amplitude, and the transition form factor for unstable $\rho \rightarrow \pi \gamma^{\star}$ was extracted as a function of photon virtuality, $Q^{2}$, in a rigorous manner from the residue of the $\rho$ pole singularity in the complex energy plane. 


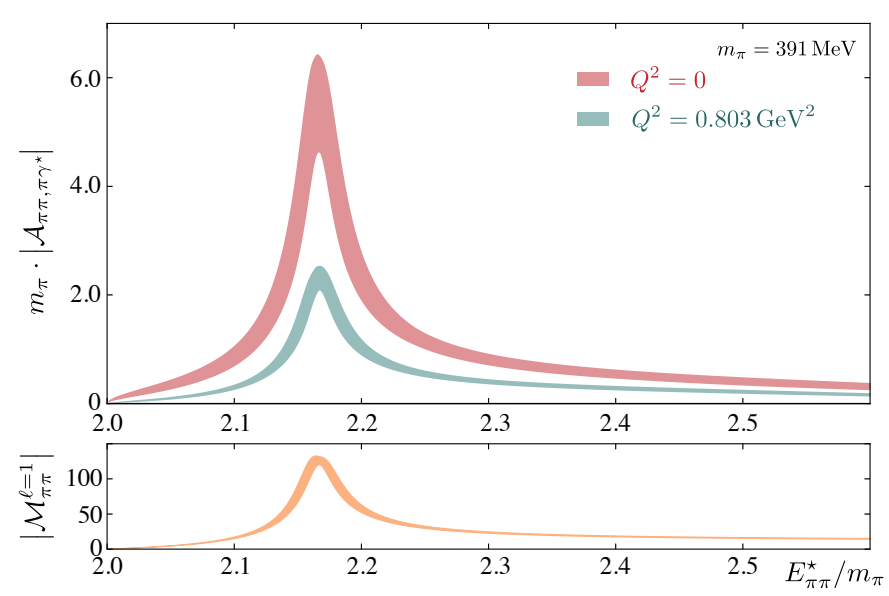

Figure 6. Upper panel: Amplitude for $\pi \gamma^{\star} \rightarrow \pi \pi$ in $P$-wave as a function of $\mathrm{cm}$-frame energy at two values of photon virtuality - the bump due to the $\rho$ resonance. Lower panel: the corresponding amplitude for elastic scattering $\pi \pi \rightarrow \pi \pi$. Both computed on a $20^{3}$ lattice with $m_{\pi}=391 \mathrm{MeV}$. Figure taken from [28].

\section{Outlook}

Combining carefully constructed operators with efficient correlation construction technology and variational analysis techniques, it has proven possible to extract large numbers of discrete excited states from lattice QCD calculations. Novel approaches have been applied to extracting scattering amplitudes from these spectra, particularly in the coupled-channel case where unitarity preserving parameterizations are required. Non-resonant and resonant cases have been considered, and recently we have seen the important extension to the case of coupling to external currents, opening up the possibility of considering photo/electro-production amplitudes, weak meson decays and other more exotic situations.

Ongoing calculations within the Hadron Spectrum Collaboration include meson-meson scattering systems featuring charmed mesons [29, 30], as well as other coupled-channel systems in the light quark sector such as $\pi \eta, K \bar{K}$ in which the $a_{0}$ resonance appears. Obvious future extensions of these calculations include systems with baryons, e.g. $N^{\star}$ resonances in $\pi N$ scattering, as well as other radiative transitions, including those between charmonium resonances [31, 32] which are well measured experimentally. A formalism to deal with three-hadron scattering amplitudes will be required to study higher-lying resonances, and progress is being made in developing the appropriate theory [33-36].

\section{Acknowledgements}

I am indebted to my colleagues within the Hadron Spectrum Collaboration, in particular Raul Briceno, Robert Edwards, Christian Shultz, Christopher Thomas and David Wilson, without whose hard work and original ideas the results presented above could not have been obtained. I am supported by the US Department of Energy through Early Career award contract DE-SC0006765 and contract DE-AC05-06OR23177, under which Jefferson Science Associates, LLC, manages and operates Jefferson Laboratory.

\section{References}

[1] M. Luscher, Nucl.Phys. B354, 531 (1991)

[2] K. Rummukainen, S.A. Gottlieb, Nucl.Phys. B450, 397 (1995), hep-lat/9503028

[3] C. Kim, C. Sachrajda, S.R. Sharpe, Nucl.Phys. B727, 218 (2005), hep-lat/0507006 
[4] Z. Fu, Phys.Rev. D85, 014506 (2012), 1110.0319

[5] L. Leskovec, S. Prelovsek, Phys.Rev. D85, 114507 (2012), 1202 . 2145

[6] S. He, X. Feng, C. Liu, JHEP 0507, 011 (2005), hep-lat/0504019

[7] M.T. Hansen, S.R. Sharpe, Phys.Rev. D86, 016007 (2012), 1204.0826

[8] R.A. Briceno, Z. Davoudi, Phys.Rev. D88, 094507 (2013), 1204 . 1110

[9] P. Guo, J. Dudek, R. Edwards, A.P. Szczepaniak, Phys.Rev. D88, 014501 (2013), 1211.0929

[10] J.J. Dudek, R.G. Edwards, M.J. Peardon, D.G. Richards, C.E. Thomas, Phys.Rev.Lett. 103, 262001 (2009), 0909.0200

[11] J.J. Dudek, R.G. Edwards, M.J. Peardon, D.G. Richards, C.E. Thomas, Phys.Rev. D82, 034508 (2010), 1004.4930

[12] C.E. Thomas, R.G. Edwards, J.J. Dudek, Phys.Rev. D85, 014507 (2012), 1107.1930

[13] J.J. Dudek, R.G. Edwards, C.E. Thomas, Phys.Rev. D86, 034031 (2012), 1203.6041

[14] M. Peardon et al. (Hadron Spectrum), Phys.Rev. D80, 054506 (2009), 0905.2160

[15] C. Michael, Nucl.Phys. B259, 58 (1985)

[16] M. Luscher, U. Wolff, Nucl.Phys. B339, 222 (1990)

[17] B. Blossier, M. Della Morte, G. von Hippel, T. Mendes, R. Sommer, JHEP 0904, 094 (2009), Q902. 1265

[18] J.J. Dudek, R.G. Edwards, C.E. Thomas (Hadron Spectrum), Phys.Rev. D87, 034505 (2013), 1212.0830

[19] K. Olive et al. (Particle Data Group), Chin.Phys. C38, 090001 (2014)

[20] J. Nebreda, J. Pelaez., Phys.Rev. D81, 054035 (2010), 1001. 5237

[21] D.J. Wilson, R.A. Briceno, J.J. Dudek, R.G. Edwards, C.E. Thomas (2015), 1507.02599

[22] J.J. Dudek, R.G. Edwards, C.E. Thomas, D.J. Wilson (Hadron Spectrum), Phys.Rev.Lett. 113, 182001 (2014), 1406.4158

[23] D.J. Wilson, J.J. Dudek, R.G. Edwards, C.E. Thomas, Phys.Rev. D91, 054008 (2015), 1411.2004

[24] L. Lellouch, M. Luscher, Commun. Math. Phys. 219, 31 (2001), hep-1at/0003023

[25] R.A. Briceno, M.T. Hansen (2015), 1502.04314

[26] R.A. Briceno, M.T. Hansen, A. Walker-Loud, Phys.Rev. D91, 034501 (2015), 1406.5965

[27] C.J. Shultz, J.J. Dudek, R.G. Edwards, Phys. Rev. D91, 114501 (2015), 1501.07457

[28] R.A. Briceno, J.J. Dudek, R.G. Edwards, C.J. Shultz, C.E. Thomas, D.J. Wilson (2015), 1507.06622

[29] G. Moir, M. Peardon, S.M. Ryan, C.E. Thomas, L. Liu, JHEP 1305, 021 (2013), 1301. 7670

[30] L. Liu, G. Moir, M. Peardon, S.M. Ryan, C.E. Thomas, P. Vilaseca, J.J. Dudek, R.G. Edwards, B. Joo, D.G. Richards (Hadron Spectrum), JHEP 07, 126 (2012), 1204 . 5425

[31] J.J. Dudek, R.G. Edwards, D.G. Richards, Phys. Rev. D73, 074507 (2006), hep-ph/0601137

[32] J.J. Dudek, R. Edwards, C.E. Thomas, Phys. Rev. D79, 094504 (2009), 0902 . 2241

[33] K. Polejaeva, A. Rusetsky, Eur.Phys.J. A48, 67 (2012), 1203. 1241

[34] R.A. Briceno, Z. Davoudi, Phys.Rev. D87, 094507 (2012), 1212. 3398

[35] M.T. Hansen, S.R. Sharpe (2015), 1504.04248

[36] M.T. Hansen, S.R. Sharpe, Phys.Rev. D90, 116003 (2014), 1408. 5933 